\title{
Correction to: Vitamin D receptor expression in peripheral blood mononuclear cells is inversely associated with disease activity and inflammation in lupus patients
}

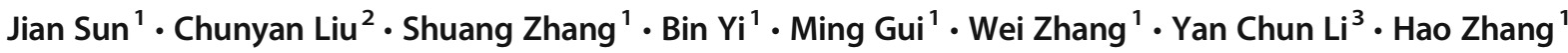 \\ Published online: 17 June 2019 \\ (C) International League of Associations for Rheumatology (ILAR) 2019
}

\section{Correction to: Clinical Rheumatology}

https://doi.org/10.1007/s10067-019-04594-2

The original version of this article, unfortunately contained an error.

The publisher regret that Funding statement was incomplete in the original publication; the authors have requested that the below Funding statement be noted.

Funding sources This study was funded by the National Natural Science Foundation of China (No. 81470961, 81870498 and 81870499 ) and the Natural Science Foundation of Hunan Province (No. 14JJ2037 and 2019JJ40463).

Publisher's note Springer Nature remains neutral with regard to jurisdictional claims in published maps and institutional affiliations.

The online version of the original article can be found at https://doi.org/ 10.1007/s10067-019-04594-2

Hao Zhang

zhanghaoliaoqing@163.com

1 Department of Nephrology and Rheumatology, The Third Xiangya Hospital, Central South University, 138 Tongzipo Road,

Changsha 410013, Hunan Province, China

2 Department of Pathology, Hunan University of Chinese Medicine, Changsha 410208, Hunan, China

3 Department of Medicine, Division of Biological Sciences, The University of Chicago, Chicago, IL 60637, USA 